\title{
Contactless Damage Evaluation of a Rotating Carbon Fiber Propeller
}

\author{
Veira Canle, Daniel
}

IEEE

2019

Veira Canle , D , Mäkinen , J M K , Lassila , P , Gritsevich , M , Salmi , A \& Haeggström , E 2019 , Contactless Damage Evaluation of a Rotating Carbon Fiber Propeller . in 2019 IEEE International Ultrasonics Symposium (IUS) . IEEE International Ultrasonics Symposium , IEEE , pp. 1579-1581, IEEE International Ultrasonics Symposium , Glasgow , United Kingdom , 06/10/2019 . https://doi.org/10.1109/ULTSYM.2019.8925618

http://hdl.handle.net/10138/337241

https://doi.org/10.1109/ULTSYM.2019.8925618

unspecified

acceptedVersion

Downloaded from Helda, University of Helsinki institutional repository.

This is an electronic reprint of the original article.

This reprint may differ from the original in pagination and typographic detail.

Please cite the original version. 
(C) 2019 IEEE. Personal use of this material is permitted. Permission from IEEE must be obtained for all other uses, in any current or future media, including reprinting/republishing this material for advertising or promotional purposes, creating new collective works, for resale or redistribution to servers or lists, or reuse of any copyrighted component of this work in other works. 


\title{
Contactless Damage Evaluation of a Rotating Carbon Fiber Propeller
}

\author{
Daniel Veira Canle ${ }^{1}$, Joni Mäkinen', Petri Lassila ${ }^{1}$, Maria Gritsevich ${ }^{1,2}$, Ari Salmi ${ }^{1}$ Edward \\ Hæggström'. \\ ${ }^{1}$ Electronics Research Laboratory, Physics Department, University of Helsinki, Finland \\ 2Institute of Physics and Technology, Ural Federal University, Mira str. 19, 620002 Ekaterinburg, \\ Russia
}

\begin{abstract}
There is a need for contactless health evaluation of curved propellers ranging from servicing drones to wind power plants. Structures that close upon themselves feature multiple paths for acoustic waves to travel which causes interference and reduces contrast. In addition, blade curvature makes it challenging to scan the structure in a contactless manner due to the difficulty to aim the laser beam. We introduce a technique that allows to scan a sample with a highly directed acoustic field. We show the feasibility of this approach by locating damage on a commercial drone propeller rotating at $1000 \mathrm{rpm}$.
\end{abstract}

\section{INTRODUCTION}

Propellers are common in drones and power plants. While functioning they are subject to stress and impacts which shortens their life span. Therefore, these components are periodically inspected to monitor their integrity [1][2][3].

The shape of these structures has been optimized for the application at hand e.g. to produce lift or harvest mechanical energy. Since they are curved, conventional ultrasonic inspection by contacting transducers is cumbersome. These transducers must be mechanically coupled into the structure to achieve efficient acoustic energy transfer. The high spatial resolution required for damage inspection would require many transducers. These transducers would load the propeller modifying its dynamic properties. Hence in situ inspection during operation is extremely challenging.

Laser ultrasound (LU) is a way to perform NDE on propellers in a contactless manner [4]. In LU the sample itself is the transducer which allows for efficient energy coupling. The use of an interferometer for acoustic wave detection means that the structure can be rapidly scanned with high spatial resolution. Pelivanov et al. proved these points by locating impact damage in a carbon reinforced polymer laminate [5]. The method they introduced rivals in spatial resolution with X-ray tomography, allowing damage detection from the opposite side of the structure where the impact is located.

Both contacting transducers and LU generate guided acoustic waves in the structure. These waves propagate long distances with little attenuation and interact with flaws in the structure such as holes and delaminations [6][7][8].

The generation of guided waves and their detection by LU is challenging in curved geometries. The curvature deflects the laser from the interferometer making the laser alignment difficult. Furthermore, embracing structures provide multiple paths for sound to travel causing interference and loss of contrast in NDE analysis [9].

We propose a method where a directed acoustic field generated by a cylindrical lens is used to scan the examined rotating structure. The rotating line allows for scanning of the blade with guided waves without the need to move the excitation or detection lasers.

\section{METHODS}

To produce a directed acoustic field, we focused an Nd:YAG laser (CFR Big Sky Laser Series, 8 ns, 3 Hz PRF 30 mJ) with a cylindrical lens featuring $80 \mathrm{~mm}$ focal length. This lens was attached to a custom-built rotational stage that moved in steps of 0.9 degrees. Due to the symmetry of the acoustic field generated by a line source, it was sufficient to rotate the line $90^{\circ}$ for every scan.

A laser Doppler vibrometer (Polytech OFV-505) in displacement mode ( $2 \mathrm{MHz}$ bandwidth, $50 \mathrm{~nm} / \mathrm{V}$ ) detected the propagating waves. In all measurements the LDV was positioned on the opposite side of the sample with respect to the excitation (Fig. 1). The location of the pick-up point was at the center of the excitation line when studying the aluminum blade. The drone propeller required positioning the detection laser on the flat edge in order to have light reflected back into the interferometer.
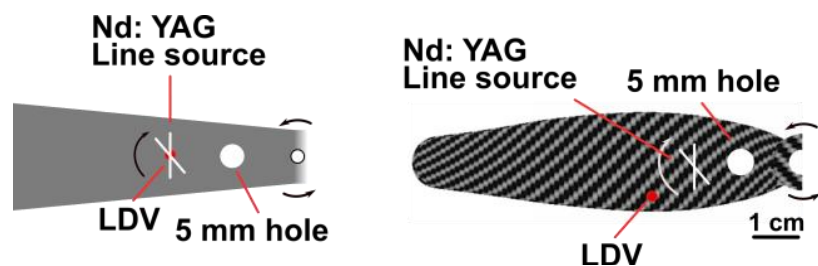

Fig. 1. Laser ultrasound experimental setup. The aluminum blade (left) allowed us to validate this technique for damage detection. Due to the curvature of the propeller blade (right) we decided to place the detection spot on the flat edge where LDV aiming is easier. The curved arrow next to the line source indicates the rotation direction of the excitation (clockwise).

To determine the phase velocity of the fastest $A_{0}$ mode we performed a linear scan on the propeller following the 
procedure described in our previous publication [4]. We fixed the detection point at $15 \mathrm{~mm}$ from the center of the carbon fiber propeller and the excitation scanned it lengthwise starting from the far edge of the blade in steps of $0.26 \mathrm{~mm}$.

We have examined two samples. A bow-tie shaped aluminum blade $\left(120 \times 40 \times 1.4 \mathrm{~mm}^{3}\right)$ and a carbon fiber drone propeller $\left(70 \times 15 \times 0.5 \mathrm{~mm}^{3}\right)$ (Hobbyking, generic) The angular velocity of these samples was adjustable by a commercial ESC motor controller (YEP ESC 150A). A custom-made optical gate based on the PDB-C142 photodiode detected the passing of the target. An Arduino Mega 2560 synchronized the measurement and a Picoscope 3000 series $(50 \mathrm{MHz}$ bandwidth) sent data to a computer. We high-pass filtered the data using a Butterworth infinite impulse response filter with a pass frequency of $100 \mathrm{kHz}$ and a cut-off frequency of 300 $\mathrm{kHz}$.

We validated the proposed technique on an aluminum blade (Figs. 2\&3). For comparison, we performed a scan on a pristine aluminum sample. Later, we drilled a $5 \mathrm{~mm}$ hole on the structure at $6.1 \mathrm{~mm}$ from the detection point and repeated the experiments (Table 1). Finally, we studied a drone propeller featuring a $5 \mathrm{~mm}$ hole both stationary and at 1000 rpm (Fig. 3).

We determined the location of the defects present on the structures by locating the first peak of the echo that radiates from the hole. The distance to the defect is then:

$\mathrm{d}=\frac{\Delta \mathrm{t}_{\mathrm{echo}}}{2} \mathrm{v}_{\mathrm{A}_{0}}$

where $\Delta \mathrm{t}_{\text {echo }}$ is the time difference between the time-of-flight of the echo and that of the ballistic $A_{0}$ mode whereas $v_{A 0}$ is the phase velocity of the antisymmetric mode.

Knowing that the defect has a circular shape, one can estimate its radius as:

$\mathrm{R}=\frac{\tan (\alpha / 2)}{1-\tan (\alpha / 2)} \mathrm{d}$, [2]

where $R$ is the defect radius, $d$ the distance from the excitation to the pick-up point and $\alpha$ the half-angle encompassing the $\mathrm{A}_{0}$ echo. To determine this angle, we integrated the amplitude of the acoustic wave squared and found the angles for which it falls by $-3 \mathrm{~dB}$. The angle is then estimated to be the difference between those boundaries.

\section{Results}
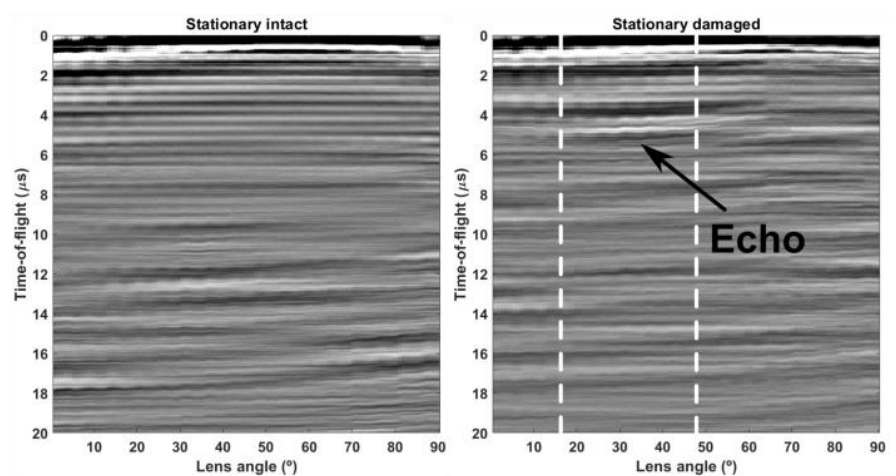

Fig. 2. Acoustic field generated by a rotating line source on a stationary aluminum blade. The presence of a defect (right) produces an echo arriving at $3.2 \mu \mathrm{s}$ compared to the intact sample (left). The width of the defect is represented by the $-3 \mathrm{~dB}$ boundaries (dashed lines).
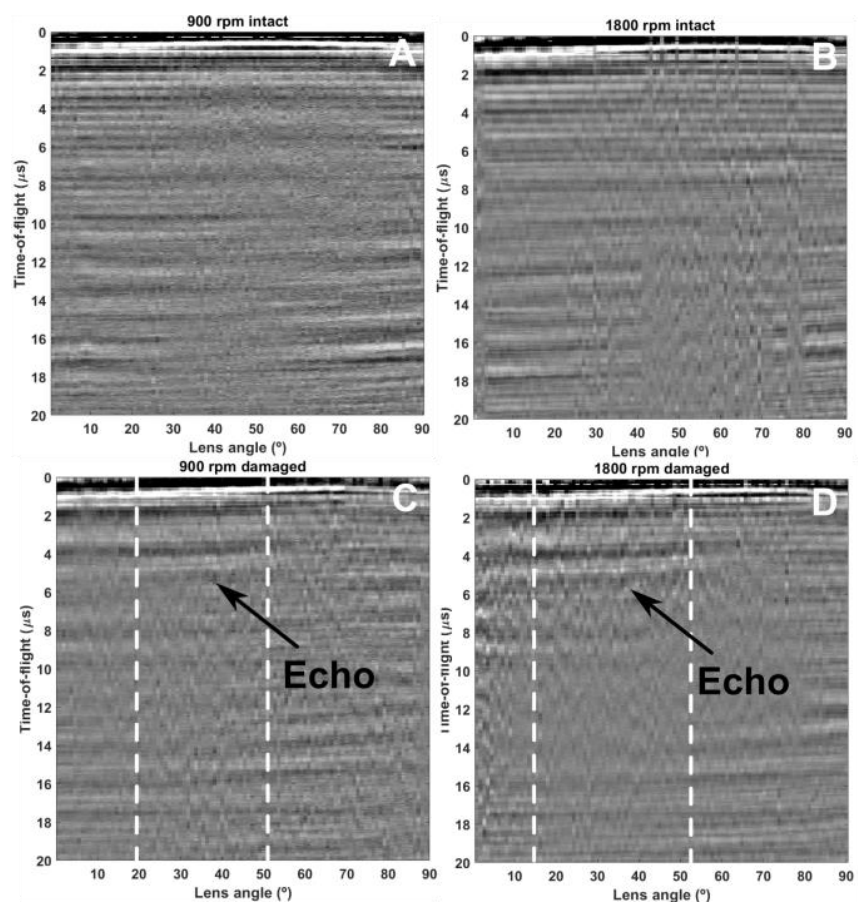

Fig. 3. Acoustic field generated by a rotating line on a rotating aluminum propeller. A, B (C, D) intact (damaged) propeller rotating at $900 \mathrm{rpm}$ and $1800 \mathrm{rpm}$, respectively.

From the previous study [4], we know the phase velocity of the $A_{0}$ mode on the aluminum structure: $3180 \pm 32 \mathrm{~ms}^{-1}$. From the linear scan of the carbon fiber propeller, the phase velocity of the quasi-A0 mode on the propeller is $1822 \pm$ $98 \mathrm{~ms}^{-1}$. 

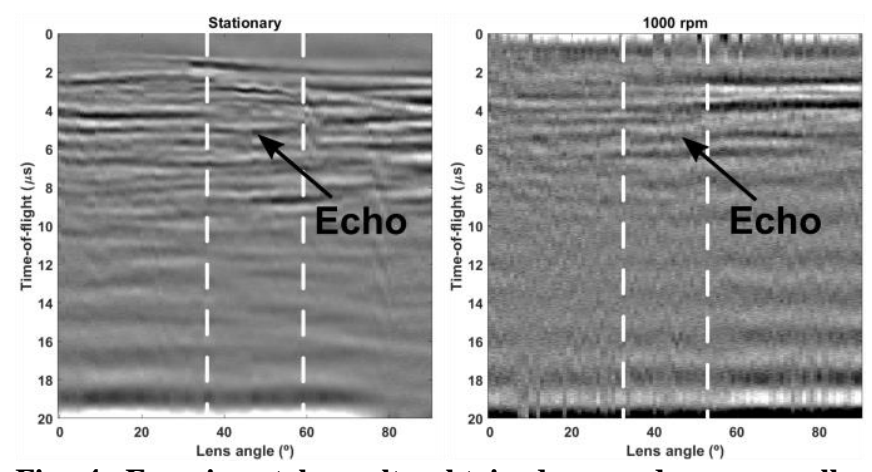

Fig. 4. Experimental results obtained on a drone propeller featuring a $5 \mathrm{~mm}$ defect stationary (left) and at $1000 \mathrm{rpm}$ (right). The dashed lines are a guide to the eye to highlight the width of the defect. The bright features at $0 \mu s$ are artifacts from high-pass filtering the data.

\begin{tabular}{|l|l|l|}
\hline & Distance $(\mathrm{mm})$ & Radius $(\mathrm{mm})$ \\
\hline Stationary & $5.25 \pm 0.46$ & $2.62 \pm 0.39$ \\
\hline $900 \mathrm{rpm}$ & $5.93 \pm 0.46$ & $2.47 \pm 0.39$ \\
\hline $1800 \mathrm{rpm}$ & $5.03 \pm 0.46$ & $2.52 \pm 0.39$ \\
\hline Measured value & $5.60 \pm 0.05$ & $2.50 \pm 0.05$ \\
\hline
\end{tabular}

Table 1. Experimental results for the aluminum blade. Here the distance is the path travelled from the defect to the detection point.

\section{Discussion}

With the drone propeller, we attempted the same approach as with the aluminum propeller. In practice we had, due to its curvature, to place the interferometer at an angle. When measuring a moving target in this configuration $2.5 \mathrm{MHz}$ frequency noise saturated the data. We hypothesize that the interferometer readings are unstable when measuring rotating curved structures at an angle. The device cannot compensate for the rapid change in curvature, thus resulting in a change of phase of the reflected light. We found that having the LDV resting on a horizontal position and aiming at the flattest part in the structure yielded clean data. This approach revealed that it was not feasible to have the detection point at the center of the rotating line. Consequently, the analysis of the propagating waves became non-trivial and we could not retrieve the distance to the defect and its size.

In this study, we limited our attention to the propagation of the $\mathrm{A}_{0}$ Lamb wave mode. This is due to the fact that the LDV measures out-of-plane displacement. Due to the shape of the $\mathrm{S}_{0}$ and $\mathrm{A}_{0}$ modes at any given time the antisymmetric wave features a larger out-of-plane displacement than the symmetric mode and thus it can be detected easily. Similar modes propagate in the composite propeller but the curvature of the structure modifies the Lamb wave modes properties, for example, their shape and cut-off frequencies [10].

Carbon fiber-based composites are anisotropic materials since they are composed of intertwined carbon fibers embedded in a polymer matrix. Since quasi-Lamb waves are a collective vibration across the thickness of the sample, the results obtained from the measurements on the composite are comparable to those performed on the aluminum blade. However, as seen in Figs. 1, 2 and 3, attenuation is much greater in the composite while in the aluminum structure waves propagate greater distances.

\section{CONCLUSION}

We introduced a laser ultrasound method that allows location and sizing of circular holes by means of directed acoustic fields on rotating structures.

\section{REFERENCES}

[1] Y.-K. An, B. Park, and H. Sohn, "Complete noncontact laser ultrasonic imaging for automated crack visualization in a plate," Smart Mater. Struct., vol. 22, no. 2, p. 025022, Feb. 2013.

FAA, "Aircraft Propeller Maintenance,"

Washington, 2005.

B. Park, H. Sohn, C.-M. Yeum, and T. C. Truong, "Laser ultrasonic imaging and damage detection for a rotating structure," Struct. Heal. Monit. An Int. J., vol. 12, no. 5-6, pp. 494-506, Sep. 2013.

D. Veira Canle, A. Salmi, and E. Hæggström, "Noncontact damage detection on a rotating blade by Lamb wave analysis," NDT E Int., vol. 92, pp. 159166, Dec. 2017.

[5] I. Pelivanov et al., "High resolution imaging of impacted CFRP composites with a fiber-optic laserultrasound scanner," Photoacoustics, vol. 4, no. 2, pp. 55-64, Jun. 2016.

[6] C. Valle, M. Niethammer, J. Qu, and L. J. Jacobs, "Crack characterization using guided circumferential waves," J. Acoust. Soc. Am., vol. 110, no. 3, pp. 1282-1290, Sep. 2001.

[7] Z. Li, C. He, Z. Liu, and B. Wu, "Quantitative detection of lamination defect in thin-walled metallic pipe by using circumferential Lamb waves based on wavenumber analysis method," NDT E Int., vol. 102, pp. 56-67, Mar. 2019.

[8] S. Towfighi and T. Kundu, "Elastic wave propagation in anisotropic spherical curved plates," Int. J. Solids Struct., vol. 40, no. 20, pp. 5495-5510, Oct. 2003.

[9] J. Mäkinen, D. Veira Canle, R. Blomqvist, A. Salmi, and E. Haeggström, "Simulation of Laser-excited Surface Acoustic Waves Traveling on A Hemispherical Steel Shell," 2018.

[10] Ka Lok Jimmy Fong, "A Study of Curvature Effects on Guided Elastic Waves," Imperial College London, 2005. 